\title{
COHOMOLOGY AND EXTENSIONS OF ORDERED GROUPOIDS
}

\author{
B. O. BAINSON AND N. D. GILBERT
}

\author{
School of Mathematical and Computer Sciences \\ and the Maxwell Institute for the Mathematical Sciences, \\ Heriot-Watt University, Edinburgh EH14 4AS, U.K.
}

\begin{abstract}
We adapt and generalise results of Loganathan on the cohomology of inverse semigroups to the cohomology of ordered groupoids. We then derive a five-term exact sequence in cohomology from an extension of ordered groupoids, and show that this sequence leads to a classification of extensions by a second cohomology group. Our methods use structural ideas in cohomology as far as possible, rather than computation with cocycles.
\end{abstract}

\section{INTRODUCTION}

Ordered groupoids arose in the work of Ehresmann [10] on the foundations of differential geometry, as an algebraic model of a pseudogroup of transformations in which the ordered structure models restriction of domain. Groupoids as models of symmetry are discussed in [31], and ordered groupoids offer a general algebraic framework for the discussion of partial symmetry. There are close connections with the theory of inverse semigroups, since the category of inverse semigroups can be viewed as a subcategory of the category of ordered groupoids. These connections are an important theme of the book [21] by Lawson, and recent research has both generalised known results from inverse semigroups to ordered groupoids (see, for example [12, 23]), and has used ordered groupoids as a more natural setting for the development of structural ideas (see, for example, [1, 22, 20, 28]).

A cohomology theory for inverse semigroups was introduced by Lausch [18], who used it to classify extensions by an inverse semigroup $S$ of an $S$-module $\mathcal{A}$, unifying earlier results of Coudron [7] and D'Alarcao [9]. Lausch's paper introduces the category $\operatorname{Mod}_{S}$ of $S$-modules, establishes that it has enough injectives, constructs a cohomology functor from $\operatorname{Mod}_{S}$ to abelian groups, and constructs projective resolutions of a homogeneous module $\Delta \mathbb{Z}$ to compute cohomology. He then shows directly, using a 2-cocycle or factor set associated to an extension, that extensions of $\mathcal{A}$ by $S$ are classified by the cohomology group $H^{2}\left(S^{1}, \mathcal{A}^{0}\right)$ of the inverse monoid $S^{1}$.

E-mail address: bob30@hw.ac.uk, N.D.Gilbert@hw.ac.uk.

2010 Mathematics Subject Classification. Primary 20L05 ; Secondary 20J06, 18 G15.

Some of these results are presented in a different form as part of the first author's PhD thesis [3]. The support of a MACS Global Platform Studentship from Heriot-Watt University is gratefully acknowledged. 
Loganathan [24] recognised that Lausch's category $\operatorname{Mod}_{S}$ could be obtained as the category of modules of a left-cancellative category $\mathrm{七}(S)$ derived from $S$ (and that $\mathrm{€}(S)$ was equivalent to an earlier construction of Leech [19]). Loganathan then developed a number of results about the homology and cohomology of $\mathrm{E}(S)$, particularly concerning its relationship to the group (co)homology of the maximal group image $\hat{S}$ of $S$, and the cohomology of the semilattice $E(S)$ of idempotents of $S$. In a subsequent paper [25] (in the more general setting of regular semigroups), Loganathan recovers Lausch's classification of extensions, again using an essentially computational approach based on cocycles. In his $\mathrm{PhD}$ thesis, J. Matthews [27] returned to Lausch's approach, to establish a cohomology theory for arbitrary ordered groupoids and to extend the use of factor sets to classify extensions. The resulting computational complications are considerable, but are well-handled in [27].

Our aim in this paper is to generalise Loganathan's account of the cohomology of inverse semigroups [24] to ordered groupoids, and to give a conceptual approach to the classification of extensions that does not involve computation with cocycles. This is carried out for groups, for example, in [17, section 6.10]. We shall adapt the account given by Gruenberg in [14, 15]. After a review in section 1 of the basic facts about ordered groupoids that we need, we turn in sections 2 and 3 to modules over ordered groupoids and ordered groupoid cohomology. These two sections are closely based on [24], and in them we check that Loganathan's constructions of some adjoint functors for modules over inverse semigroups can be generalised to ordered groupoids, and that key identifications of cohomology groups with certain Ext-groups remain valid. Apart from these generalisations and adaptations, we make no claim of any originality in these two sections, although we do proffer some additional detail omitted in [24]. In section 4 we discuss extensions of ordered groupoids involving an identity-separating map. Our main result (Theorem 4.9) is the construction of a five-term exact sequence in cohomology, which is obtained using an intermediate construction of a derived module of a functor between ordered groupoids, a generalisation of Crowell's derived module of a group homomorphism [8]. The five-term exact sequence is then the principal component in the classification of extensions by $H^{2}\left(Q^{I}, \mathcal{A}^{0}\right)$ in section 5. A crucial step is to show that, up to equivalence, any extension of a $Q$-module $\mathcal{A}$ by an ordered groupoid $Q$ can be obtained as a quotient of a semidirect product $\mathbb{F}(Q) \ltimes \mathcal{A}$, where $\mathbb{F}(Q)$ is a free ordered groupoid. Here we need to use the quotient of ordered groupoids introduced in [1], whose construction is recalled in section 1]

Related work on the cohomology of categories can be found in [29, 30, 32]. In particular, Webb [30] obtains five-term exact sequences in the (co)homology of categories. However, since the constructions in this paper originate from an ordered groupoid $G$ rather than from its associated category $\mathrm{\iota}(G)$, there are resulting differences at key points, such as in the definition of the analogue of the group ring, of the augmentation ideal, and the notion of extension.

\section{ORdERED GRoupoids}

A groupoid $G$ is a small category in which every morphism is invertible. The set of identities of $G$ is denoted by $G_{0}$, but we shall sometimes use $E(G)$, following the customary notation for the set of idempotents in an inverse semigroup. We write $g \in G(e, f)$ when $g$ is a morphism starting at $e$ and ending at $f$. We regard a groupoid as an algebraic structure comprising its morphisms, and compositions of morphisms as a partially defined 
binary operation (see [16], [21]). The identities are then written as $e=g \mathbf{d}=g g^{-1}$ and $f=g \mathbf{r}=g^{-1} g$ respectively. The star and costar of $G$ at an identity $e$ are defined as the sets $\operatorname{star}_{G}(e)=\left\{g \in G \mid g g^{-1}=e\right\}$ and $\operatorname{costar}_{G}(e)=\left\{g \in G \mid g^{-1} g=e\right\}$ respectively: their intersection is the local group $G_{e}$. A groupoid map $\theta: G \rightarrow H$ is just a functor: groupoids together with groupoid maps then constitute the category $\mathbf{G p d}$ of groupoids.

Definition 1.1. An ordered groupoid is a pair $(G, \leqslant)$ where $G$ is a groupoid and $\leqslant$ is a partial order defined on $G$, satisfying the following axioms:

OG1 $x \leqslant y \Rightarrow x^{-1} \leqslant y^{-1}$, for all $x, y \in G$.

OG2 Let $x, y, u, v \in G$ such that $x \leqslant y$ and $u \leqslant v$. Then $x u \leqslant y v$ whenever the compositions $x u$ and $y v$ exist.

OG3 Suppose $x \in G$ and $e \in G_{0}$ such that $e \leqslant x \mathbf{d}$, then there is a unique element $(e \mid x)$ called the restriction of $x$ to $e$ such that $(e \mid x) \mathbf{d}=e$ and $(e \mid x) \leqslant x$.

OG4 If $x \in G$ and $e \in G_{0}$ such that $e \leqslant x \mathbf{r}$, then there exist a unique element $(x \mid e)$ called the corestriction of $x$ to $e$ such that $(x \mid e) \mathbf{r}=e$ and $(x \mid e) \leqslant x$.

It is easy to see that OG3 and OG4 are equivalent: if OG3 holds then we may define a corestriction $(x \mid e)$ by $(x \mid e)=\left(e \mid x^{-1}\right)^{-1}$.

An ordered functor $\phi: G \rightarrow H$ of ordered groupoids is an order preserving groupoid-map, that is $g \phi \leqslant h \phi$ if $g \leqslant h$. Ordered groupoids together with ordered functors constitute the category of ordered groupoids, OGpd. An ordered functor $\phi: G \rightarrow H$ is identityseparating if it is injective when restricted to $G_{0}$.

Suppose $g, h \in G$ and that the greatest lower bound $\ell$ of $g \mathbf{r}$ and $h \mathbf{d}$ exist, then we define the pseudoproduct of $g$ and $h$ by $g * h=(g \mid \ell)(\ell \mid h)$. An ordered groupoid is called inductive if the pair $\left(G_{0}, \leqslant\right)$ is a meet semilattice. In an inductive groupoid $G$, the pseudoproduct is everywhere defined and $(G, *)$ is then an inverse semigroup: see [21, Theorem 4.1.8]

To any ordered groupoid $G$ we associate a category $€(G)$ as follows. The objects of $€(G)$ are precisely the objects of $G$ and morphisms are given by pairs $(e, g) \in G_{0} \times G$ where $g \mathbf{d} \leqslant e$, with $(e, g) \mathbf{d}=e$ and $(e, g) \mathbf{r}=g \mathbf{r}$. The composition of morphisms is defined by the partial product $(e, g)(f, h)=(e, g * h)=(e,(g \mid h \mathbf{d}) h)$ whenever $g \mathbf{r}=f$. It is easy to that $€(G)$ is left cancellative. This construction originates in the work of Loganathan [24], and forms the basis of the treatment in [24] of the cohomology of inverse semigroups.

Let $G$ be an ordered groupoid and set $G^{I}=G \cup\{I\}$ where $I$ is an identity and $I \notin G$. Set $e \leqslant I$ for every object $e$ of $G$. It is easy to see that $G^{I}$ is an ordered groupoid with maximal ordered subgroupoids $G$ and the singleton groupoid $\{I\}$. The associated category $€\left(G^{I}\right)$ is defined by the set of objects $E(G) \cup\{I\}$ and morphisms comprising of morphisms of $G$ and order maps $\alpha_{e}^{I}: I \rightarrow e$ for all $e \in E(G)$ inherited from the extension of the ordering on $G$.

We shall need the construction of a quotient of an ordered groupoid by a normal ordered subgroupoid given in [1, section 4].

Definition 1.2. An ordered subgroupoid $N$ of an ordered groupoid $G$ is a normal ordered subgroupoid if the following axioms are satisfied:

N01 $N$ has the same set of objects as $G$ : that is $N$ is wide.

N02 Suppose $n \in N$ and $e \leqslant n \mathbf{d}$ for $e \in G_{0}$, then the restriction $(n \mid e)$ of $n$ to $e$ is in $N$. 
N03 If $n \in N$ and $k, h \in G$ such that:

(a) $k$ and $h$ have an upper bound $g \in G$, that is $k \leqslant g$ and $h \leqslant g$,

(b) $h^{-1} n k$ exists in $G$, then $h^{-1} n k \in N$.

A normal ordered subgroupoid $N$ of an ordered groupois $G$ determines an equivalence relation $\simeq_{N}$ on $G$, defined by

$$
\begin{aligned}
g \simeq_{N} h \Longleftrightarrow & \text { there exist } a, b, c, d \in N \text { such that } a g b \text { and } c h d \text { are defined in } G, \\
& \text { with } a g b \leqslant h \text { and } c h d \leqslant g .
\end{aligned}
$$

The relation

$$
[g] \leqslant[h] \Longleftrightarrow \text { there exist } a, b \in N \text { such that } a g b \leqslant h
$$

is then a partial order on the set $G / / N$ of $\simeq_{N}$-classes. If $g^{-1} g \simeq_{N} h h^{-1}$ then there exist $p, q \in N$ such that $p p^{-1} \leqslant g^{-1} g, p^{-1} p=h h^{-1}, q^{-1} q \leqslant h h^{-1}$, and $q q^{-1}=g^{-1} g$. Then $\left(g \mid p p^{-1}\right) p h \simeq_{N} g q\left(q^{-1} q \mid h\right)$ and the composition of $\simeq_{N}$ classes given by $[g][h]=$ $\left[\left(g \mid p p^{-1}\right) p h\right]$ is well-defined and makes $G / / N$ into an ordered groupoid (see [1, Theorem 4.14]).

In the case that $N$ is a union of groups, it is easy to see that the relation $\simeq_{N}$ simplifies to

$$
g \simeq_{N} h \Longleftrightarrow \text { there exist } a, b \in N \text { such that } h=a g b .
$$

In particular, this will apply when $N$ is a $G$-module,

\section{MODULES OVER ORDERED GROUPOIDS}

We now review the theory of modules over ordered groupoids, following the approach of Loganathan in [24] for inverse semigroups.

Definition 2.1. Let $G$ be an ordered groupoid, with associated category $\mathrm{Ł}(G)$. A $G$ module $\mathcal{A}$ is a functor $\mathcal{A}: \mathrm{t}(G) \rightarrow \mathbf{A b}$ associating to each $e \in E(G)$ the abelian group $A_{e}$ and a group homomorphism $\triangleleft(e, g): A_{e} \rightarrow A_{g^{-1} g}$ for every morphism $(e, g) \in$ $\mathrm{七}(G)$. The map $\triangleleft(e, g)$ is the composite of a group homomorphism $\triangleleft\left(e, g g^{-1}\right)$ and a group isomorphism $\triangleleft\left(g g^{-1}, g\right)$. Morphisms of $G$-modules are called $G$-maps. We denote the functor category whose objects are $G$-modules and morphisms are $G$-maps by $\operatorname{Mod}_{\mathbf{L}(G)}$.

Example 2.2. Let $A$ be an abelian group. The constant module $\Delta A$ is given by $\mathcal{A}_{e}=A$ for all $e \in E(G)$, and all morphisms $\triangleleft(e, g)$ equal to the identity on $A$.

Example 2.3. The adjoint module over an ordered groupoid $G$ is defined as the functor $\mathbb{Z} G: \mathrm{t}(G) \rightarrow \mathbf{A b}$ which associates to every $e \in E(G)$ the free abelian group on $\operatorname{costar}_{G}(e)$. A morphism $g \in G(e, y)$ gives a morphism $(\mathbb{Z} G)_{e} \rightarrow(\mathbb{Z} G)_{y}$ given on basis elements $h$ of $(\mathbb{Z} G)_{e}$ by $h \mapsto h g$. For an order map $(e, f)$ we get a morphism $(\mathbb{Z} G)_{e} \rightarrow(\mathbb{Z} G)_{f}$ given on basis elements $g$ in $(\mathbb{Z} G)_{e}$ by the mapping $g \mapsto(f \mid g)$ where $(f \mid g)$ is the unique corestriction of $g$ to $f$.

Lemma 2.4. If the ordered groupoid $G$ has a maximum identity $1 \in G_{0}$ then the adjoint module is a free $G$-module (and so is projective).

Proof. A basis element $h \in(\mathbb{Z} G)_{e}$ is uniquely expressible as $1 \triangleleft(1, h)$. 
The augmentation map is the $G$-map $\mathbb{Z} G \stackrel{\epsilon}{\rightarrow} \Delta \mathbb{Z}$ defined by $\sum_{g^{-1} g=e} n_{g} g \mapsto \sum_{g^{-1} g=e} n_{g}$. Its kernel, denoted by $K G$, is called the augmentation module.

Lemma 2.5. The set of all elements $g-e$ where $g$ is a non-identity morphism in $\operatorname{costar}_{G}(e)$, forms a $\mathbb{Z}$-basis of the abelian group $(K G)_{e}$

2.1. Restriction to $G_{0}$. The set of identities $G_{0}$ is a trivial subgroupoid of $G$, and also a poset, and as such can be regarded as a category $E(G)$ in which there is a unique morphism $x \rightarrow y$ whenever $x \geqslant y$ in $G_{0}$. It is easy to see that $\mathrm{Ł}\left(G_{0}\right)$ coincides with $E(G)$. In [24], Loganathan shows that for an inverse semigroup $S$, the inclusion $E(S) \hookrightarrow \mathrm{E}(S)$ induces a restriction $\operatorname{Mod}_{\mathfrak{L}(S)} \rightarrow \operatorname{Mod}_{E(S)}$ which admits a left adjoint. This construction remains valid for ordered groupoids, as we now show. Let $\mathcal{B} \in \operatorname{Mod}_{E(G)}$ and let $B_{e}^{(g)}$ be a copy of $B_{e}$ labelled by $g \in G$. We define

$$
(\mathscr{H} \mathcal{B})_{e}=\bigoplus_{\substack{g \in G \\ g^{-1} g=e}} B_{g g^{-1}}^{(g)}
$$

Suppose $g \in G(e, y)$ and let $B_{h h^{-1}}^{h}$ be a summand of $(\mathscr{H B})_{e}$. Then there is a copy $B_{h h^{-1}}^{(h g)}$ of $B_{h h^{-1}}$ in $(\mathscr{H} \mathcal{B})_{y}$ labelled by $h g$, and so right multiplication of the labels by $g$ induces a homomorphism $\rho_{g}:(\mathscr{H B})_{e} \rightarrow(\mathscr{H B})_{y}$. Suppose that $e, f \in G_{0}$ and that $(e, f)$ is a morphism in $\mathrm{七}(G)$. Let $x \in G$ with $x^{-1} x=e$ : then $B_{x x^{-1}}^{(x)}$ is a summand of $(\mathscr{H B})_{e}$. Let $p=(x \mid f)$ d. Then $B_{p}^{((x \mid f))}$ is a summand of $(\mathscr{H B})_{f}$ and so $(e, f)$ induces a map $(\mathscr{H} \mathcal{B})_{e} \rightarrow(\mathscr{H} \mathcal{B})_{f}$. In this way, $\mathscr{H} \mathcal{B}$ is a $G$-module.

Proposition 2.6. Let $G$ be an ordered groupoid and let $\mathcal{H}$ be the functor $\operatorname{Mod}_{E(G)} \rightarrow$ $\operatorname{Mod}_{\ell(G)}$ defined above. Then $\mathscr{H}$ is left adjoint to the restriction $\operatorname{Mod}_{\ell(G)} \rightarrow \operatorname{Mod}_{E(G)}$.

Proof. Given an $E(G)$-module $\mathcal{B}$ and an $€(G)$-module $\mathcal{C}$, we obtain a natural bijection $\operatorname{Mod}_{E(G)}(\mathcal{B}, \mathcal{C}) \rightarrow \operatorname{Mod}_{\mathbf{L}(G)}(\mathscr{H} \mathcal{B}, \mathcal{C})$ as follows.

Given $\psi \in \operatorname{Mod}_{\mathfrak{(}(G)}(\mathscr{H} \mathcal{B}, \mathcal{C})$, we define $\phi \in \operatorname{Mod}_{E(G)}(\mathcal{B}, \mathcal{C})$ by setting $\phi_{e}: B_{e} \rightarrow C_{e}$ to be the restriction $\psi \mid B_{e}^{(e)}$. This obviously defines an $E(G)-$ map $\mathcal{B} \rightarrow \mathcal{C}$. On the other hand, given $\phi \in \operatorname{Mod}_{E(G)}(\mathcal{B}, \mathcal{C})$ we define $\psi \in \operatorname{Mod}_{£(G)}(\mathscr{H} \mathcal{B}, \mathcal{C})$, where

$$
\psi_{e}: \bigoplus_{\substack{g \in G \\ g^{-1} g=e}} B_{g g^{-1}} \rightarrow C_{e}
$$

by setting

$$
\psi_{e} \mid B_{g g^{-1}}^{(g)}=\alpha_{g}^{-1} \phi_{g g^{-1}} \alpha_{g} .
$$

where $\alpha_{g}$ is the action of $g \in G$ mapping $B_{g g^{-1}} \rightarrow B_{g^{-1} g}$ and $C_{g g^{-1}} \rightarrow C_{g^{-1}}$. For $e, f \in E(G)$ with $e \geqslant f$, let $\beta_{f}^{e}: B_{e} \rightarrow B_{f}$ and $\gamma_{f}^{e} ; C_{e} \rightarrow C_{f}$ be the maps giving the $E(G)$-module structure on $\mathcal{B}$ and $\mathcal{C}$ and, for $g \in G$ with $g^{-1} g=e$, consider the following diagram: 


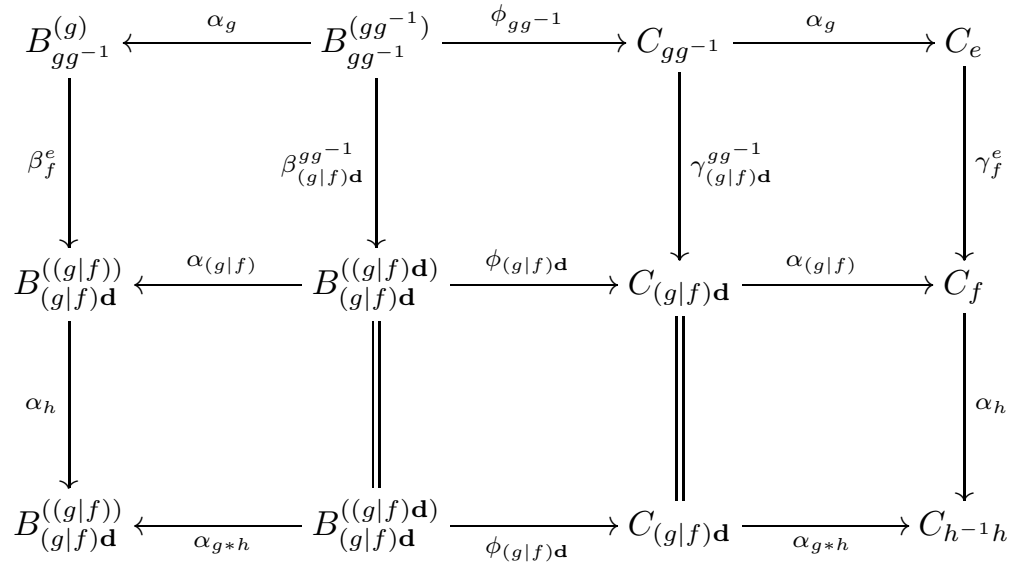

Each of the six constituent squares here is commutative for obvious reasons, and so the whole diagram commutes, and shows that $\psi$ as defined from $\phi$ above is a $G$-map. Moreover, it is easy to see that the given constructions that define $\phi$ from $\psi$ and $\psi$ from $\phi$ are inverse.

2.2. Ordered groupoids with an adjoined identity. The inclusion $G \rightarrow G^{I}$ induces a functor $\operatorname{Mod}_{\mathfrak{L}_{\left(G^{I}\right)}} \rightarrow \operatorname{Mod}_{\mathbf{\ell}(G)}$ which associates each $G^{I}$-module with the restricted module over the maximal ordered subgroupoid $G$ of $G^{I}$. We will show that this has a right adjoint . A parallel result is found in [24] for inverse monoids.

Given $\mathcal{A} \in \operatorname{Mod}_{£(G)}$ we define $\mathcal{A}^{I}$ by

$$
\mathcal{A}_{e}^{I}= \begin{cases}\mathcal{A}_{e} & \text { if } e \neq I \\ \lim ^{E(G)} \mathcal{A} & \text { if } e=I\end{cases}
$$

where $e \in E(G) \cup\{I\}$.

Lemma 2.7. Let $G$ be an ordered groupoid and $\mathcal{A} \in \operatorname{Mod}_{\ell(G)}$. Then $\mathcal{A}^{I}$ is a $G^{I}$-module.

Proof. A morphism in $\mathrm{€}\left(G^{I}\right)$ but not in $\mathrm{€}(G)$ has the form $(I, g)$ for some $g \in G$, and it is the action of such morphisms that we need to explain. Since $(I, g)=\left(I, g g^{-1}\right)\left(g g^{-1}, g\right)$ then it suffices to consider $(I, e)$ for $e \in G_{0}$ : then the map $\lim ^{E(G)} \mathcal{A} \rightarrow A_{e}$ corresponding to $(I, e) \in \mathrm{七}\left(G^{I}\right)$ is the canonical projection $\pi_{e}$. Let $\alpha_{g}: A_{g g^{-1}} \rightarrow A_{g^{-1} g}$ and $\alpha_{f}^{e}: A_{e} \rightarrow$ $A_{f}$ (where $e \geqslant f$ ) be the maps giving the $G$-module structure on $\mathcal{A}$. Then consider the following diagram, in which $x=\left(g \mid h h^{-1}\right) \mathbf{d}$ :

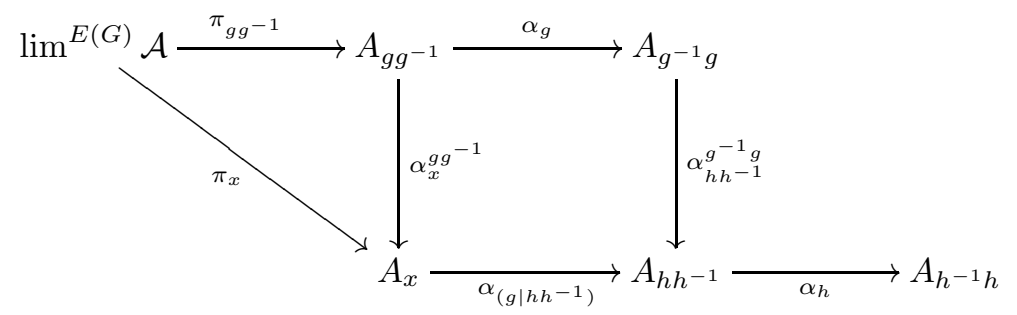


Since $\left(g g^{-1}, x\right)\left(x,\left(g \mid h h^{-1}\right)\right)=\left(g g^{-1}, g\right)\left(g^{-1} g, h h^{-1}\right) \in \mathrm{Ł}(G)$, the central square commutes, and the left-hand triangle commutes by the definition of lim. It follows that $\mathcal{A}^{I}$ is a $G$-module,

Proposition 2.8. The functor $\operatorname{Mod}_{\ell(G)} \rightarrow \operatorname{Mod}_{L_{\left(G^{I}\right)}}$ defined by $\mathcal{A} \mapsto \mathcal{A}^{I}$ is right adjoint to the restriction $\operatorname{Mod}_{\ell\left(G^{I}\right)} \rightarrow \operatorname{Mod}_{\ell(G)}$.

Proof. Let $\mathcal{B}$ be a $G^{I}$-module and let $\mathcal{C}$ be a $G$-module. We exhibit a natural bijection

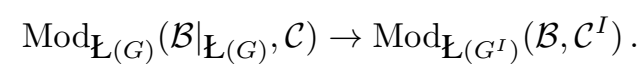

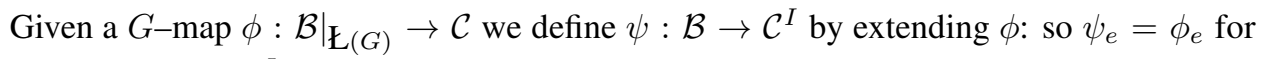
$e \neq I$ and $\psi_{I}=\beta_{e}^{I} \phi_{e}$. Since $\phi$ is a $G$-map the following diagram commutes:

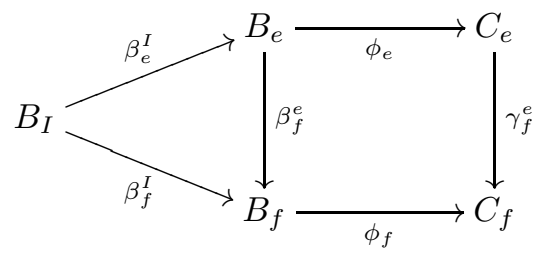

and so there exists an induced map $\psi_{I}: \mathcal{B}_{I} \rightarrow \lim ^{E(G)} \mathcal{C}$. This completes the definition of $\psi$ as a $G^{I}$-map since the diagram

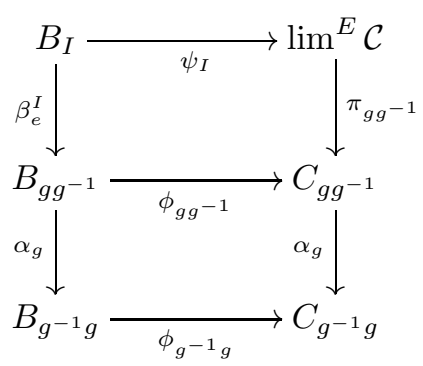

then also commutes. The correspondence $\phi \mapsto \psi$ is then easily seen to be a bijection, as required.

Let $\mathcal{A}^{0}$ be the $G^{I}$-module obtained from the $G$-module $\mathcal{A}$ by associating to $I$ the trivial group $\{0\}$. It is clear that $\mathcal{A}^{0}$ admits an $\mathrm{\complement}\left(G^{I}\right)$-action. The functor $\operatorname{Mod}_{\mathfrak{E}_{(G)}} \rightarrow$ $\operatorname{Mod}_{\mathbf{\ell}\left(G^{I}\right)}$ defined by $\mathcal{A} \mapsto \mathcal{A}^{0}$ is then left adjoint to the restriction $\operatorname{Mod}_{\mathbf{\ell}\left(G^{I}\right)} \rightarrow \operatorname{Mod}_{\mathbf{E}(G)}$.

\section{COHOMOLOGY OF ORDERED GROUPOIDS}

We now apply the concept of cohomology of small categories as discussed in [11] and [6] to ordered groupoids, still following Loganathan's approach in [24]. Suppose $\mathcal{A} \in \operatorname{Mod}_{\mathfrak{L}(G)}$. We have $\operatorname{Ext}_{\mathfrak{\complement}(G)}^{n}(\Delta \mathbb{Z}, \mathcal{A})=H^{n}\left(\operatorname{Mod}_{\mathfrak{\complement}(G)}(P, \mathcal{A})\right)$ where $P$ is a projective resolution of $\Delta \mathbb{Z}$. The cohomology of $G$ with coefficients in $\mathcal{A}$ is defined by

$$
H^{n}(G, \mathcal{A})=\operatorname{Ext}_{\mathfrak{L}(G)}^{n}(\Delta \mathbb{Z}, \mathcal{A}) .
$$

Properties of Ext as the derived functor of lim then give the following result. 
Proposition 3.1. Let $G$ be an ordered groupoid and $\mathcal{A} \in \operatorname{Mod}_{\ell(G)}$. Then

(1) $H^{0}(G, \mathcal{A})=\lim ^{\ell(G)} \mathcal{A}$.

(2) $H^{n}(G, \mathcal{A})=0$ for $n>0$ and $\mathcal{A}$ injective.

Theorem 3.2. Suppose $G$ is an ordered groupoid and let $\mathcal{A}$ be a $G$-module. Then there are natural isomorphisms $H^{n}(E(G), \mathcal{A}) \cong \operatorname{Ext}_{\perp(G)}^{n}(\mathbb{Z} G, \mathcal{A})$.

Proof. The proof is adapted fom [24]. The category $\operatorname{Mod}_{E(G)}$ has enough projectives and so every $E(G)$-module admits a projective resolution. Choose an $E(G)$-projective resolution $P$ of $\Delta \mathbb{Z}$. The functor $\mathscr{H}$ from Proposition 2.6 is left adjoint to restriction and so preserves projectives: hence $\mathscr{H} P$ is a projective resolution of $\mathscr{H} \Delta \mathbb{Z}=\mathbb{Z} G$. Then

$$
\begin{aligned}
H^{n}(E(G), \mathcal{A})=\operatorname{Ext}_{E(G)}^{n}(\Delta \mathbb{Z}, \mathcal{A}) & =H^{n}\left(\operatorname{Mod}_{E(G)}(P, \mathcal{A})\right) \\
& =H^{n}\left(\operatorname{Mod}_{\mathfrak{L}(G)}(\mathscr{H} P, \mathcal{A})\right) \\
& =\operatorname{Ext}_{\mathfrak{\complement}(G)}^{n}(\mathscr{H} \Delta \mathbb{Z}, \mathcal{A})=\operatorname{Ext}_{\mathfrak{\complement}(G)}^{n}(\mathbb{Z} G, \mathcal{A}) .
\end{aligned}
$$

Suppose $G^{I}$ is an ordered groupoid obtained from $G$ by adjoining the identity $I$ and consider the augmentation modules $K G$ and $K G^{I}$.

Lemma 3.3. For $n \geqslant 0$, there are canonical isomorphisms,

$$
\operatorname{Ext}_{\ell\left(G^{I}\right)}^{n}\left(K G^{I}, \mathcal{A}^{0}\right) \cong \operatorname{Ext}_{\ell\left(G^{I}\right)}^{n}\left((K G)^{0}, \mathcal{A}^{0}\right) \cong \operatorname{Ext}_{\ell(G)}^{n}(K G, \mathcal{A}) .
$$

Proof. The augmentation module $K G^{I}$ satisfies $\left(K G^{I}\right)_{e}=(K G)_{e}$ for $e \neq I$, and

$$
\left(K G^{I}\right)_{I}=\operatorname{ker}\left(\left(\mathbb{Z} G^{I}\right)_{I} \rightarrow \mathbb{Z}\right)=\operatorname{ker}(\mathbb{Z} \rightarrow \mathbb{Z})=\{0\} .
$$

Therefore $K G^{I}=(K G)^{0}$, and the result follows.

Theorem 3.4. Let $\mathcal{A}$ be a $G$-module. Then, for $n>0$, we have

$$
H^{n}\left(G^{I}, \mathcal{A}^{0}\right) \cong \operatorname{Ext}_{亡(G)}^{n-1}(K G, \mathcal{A}) .
$$

Proof. Using (3.1) and Lemma 3.3, the long exact Ext-sequence obtained from the short exact sequence $0 \rightarrow K G^{I} \rightarrow \mathbb{Z} G^{I} \rightarrow \Delta \mathbb{Z} \rightarrow 0$ of $G^{I}$-modules is

$$
\begin{aligned}
\cdots \rightarrow H^{n}\left(G^{I}, \mathcal{A}^{0}\right) & \rightarrow \operatorname{Ext}_{€}^{n}\left(G^{I}\right) \\
& \left.\rightarrow \mathbb{Z}^{n+1}\left(G^{I}, \mathcal{A}^{0}\right) \rightarrow \mathcal{A}^{0}\right) \rightarrow \operatorname{Ext}_{\mathrm{Ex}_{\left(G^{I}\right)}^{n+1}}^{n}\left(\mathbb{Z} G^{I}, \mathcal{A}^{0}\right) \rightarrow \cdots
\end{aligned}
$$

and for $n=0$ we have

$$
H^{0}\left(E\left(G^{I}\right), \mathcal{A}^{0}\right)=\operatorname{Ext}_{\mathfrak{(}\left(G^{I}\right)}^{0}\left(\mathbb{Z} G^{I}, \mathcal{A}^{0}\right)=\operatorname{Mod}_{\mathfrak{(}\left(G^{I}\right)}\left(\mathbb{Z} G^{I}, \mathcal{A}^{0}\right) .
$$

Consider a basis element $g \in\left(Z G^{I}\right)_{e}$, with $g^{-1} g=e$ : then $g=I \triangleleft(I, g)$, and so for any $G^{I}$-map $\psi: \mathbb{Z} G^{I} \rightarrow \mathcal{A}^{0}$, we have

$$
g \psi_{e}=(I \triangleleft(I, g)) \psi_{e}=\left(I \psi_{I}\right) \triangleleft(I, g)=0
$$

since $\left(\mathcal{A}^{0}\right)_{I}=\{0\}$. Hence $\psi$ is trivial, and $\operatorname{Mod}_{\mathfrak{Ł}_{\left(G^{I}\right)}}\left(\mathbb{Z} G^{I}, \mathcal{A}^{0}\right)=\{0\}$. From this, and the fact that $\mathbb{Z} G^{I}$ is projective by Lemma2.4, it follows that $\operatorname{Ext}_{\left.\mathfrak{(} G^{I}\right)}^{n}\left(\mathbb{Z} G^{I}, \mathcal{A}^{0}\right)=0$ for all $n \geqslant 0$. Therefore, the long exact sequence (3.2) splits into the isomorphisms claimed in the Theorem. 


\section{EXTENSIONS OF ORDERED GROUPOIDS}

Our aim, in the remainder of the paper, is to show how extensions of ordered groupoids, with quotient $Q$ and kernel a $Q$-module $\mathcal{A}$, are classifed by the second cohomology group $H^{2}\left(Q^{I}, \mathcal{A}^{0}\right)$. This extension theory has appeared in [27] as a generalisation of that developed by Lausch in [18] for inverse semigroups. We shall give a simpler, more conceptual account which is based on the approach due to Gruenberg in [15] for group extensions. The main ingredient is a five-term exact sequence in cohomology, associated to an extension, and we shall now proceed to derive this. The corresponding five-term exact sequence in group cohomology is well-known and can be found, for example, in [17, section VI.8].

Working in the category of inverse semigroups, Lausch [18] defined an extension of a semilattice of abelian groups $\mathcal{A}$ by an inverse semigroup $S$ to be an inverse semigroup $U$ containing $\mathcal{A}$, with an idempotent-separating homorphism $\psi: U \rightarrow S$ such that $\mathcal{A}=\{u \in$ $U: u \psi \in E(S)\}$. This definition was extended, in the natural way, to ordered groupoids by Matthews [27]. We shall need a more general version, omitting the requirement that we have a semilattice of abelian groups.

Definition 4.1. Let $Q$ be an ordered groupoid, and let $N$ be an ordered groupoid that is a disjoint union of groups $N_{x}, x \in N_{0}$. An extension of $N$ by $Q$ is an ordered groupoid $G$ together with an ordered embedding $N \stackrel{\iota}{\rightarrow} G$ and an identity-separating surjective ordered functor $\phi: G \rightarrow Q$ such that $N$ is the kernel of $\phi$ : that is, $N=\left\{g \in G: g \phi \in Q_{0}\right\}$.

We note some simple consequences of this definition (as in [27, Proposition10.1]).

Lemma 4.2. Let $N \stackrel{\iota}{\rightarrow} G \stackrel{\phi}{\rightarrow} Q$ be an extension of $N$ by $Q$. Then

(a) $N$ is a normal ordered subgroupoid of $G$,

(b) there is an isomorphism $G / / N \rightarrow Q$ of ordered groupoids, mapping $[g]_{N} \mapsto g \phi$.

Two extensions $G$ and $H$ of $N$ by $Q$ are equivalent if there exists an ordered functor $\mu$ making the following diagram commute:

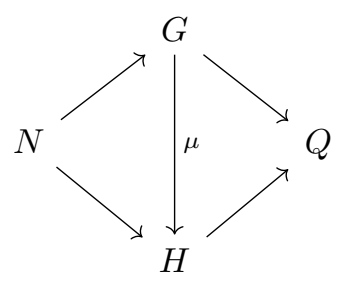

Given a $Q$-module $\mathcal{A}$ we can define the semidirect product or action groupoid $Q \ltimes A$ (as defined more generally by Brown [4] in the unordered case, and by Steinberg [28] for ordered groupoids) which will be an extension of $\mathcal{A}$ by $Q$. The set of arrows of $Q \ltimes A$ is $\left\{(q, a): a \in A_{q \mathbf{r}}\right\}$, the set of objects is $Q_{0}$, and the domain and range maps are defined by $(q, a) \mathbf{d}=q \mathbf{d}$ and $(q, a) \mathbf{r}=q \mathbf{r}$. The composition is

$$
(g, a)(h, b)=(g h,(a \triangleleft h)+b)
$$

defined when $g \mathbf{r}=h \mathbf{d}$, and the ordering is componentwise. 
4.1. Five-term exact sequences. The preliminary step in constructing the five-term exact sequence is to assign a short exact sequence of modules to an extension of ordered groupoids. This is a generalisation of the ideas of Crowell in [8] for groups. Our approach is adapted from the account in [5] for groupoids that are trivially ordered.

The following definitions are taken from [2] and generalise definitions given for inverse semigroups in [13].

Definition 4.3. Suppose that $G \stackrel{\ominus}{\rightarrow} Q$ is an ordered morphism of ordered groupoids and let $\mathcal{B}$ be a $Q$-module. Then an order-preserving function $f: G \rightarrow \mathcal{B}$ is called an ordered $\theta$-derivation if:

(1) $g f \in \mathcal{B}_{\left(g^{-1} g\right) \theta}$

(2) $(g h) f=(g f \triangleleft h \theta)+h f$, whenever $g h$ is defined in $G$.

The set $\operatorname{Der}_{\theta}(G, \mathcal{B})$ of all $\theta$-derivations $G \rightarrow \mathcal{B}$ is an abelian group under pointwise addition. If $\theta=\operatorname{id}_{G}$ we just write $\operatorname{Der}(G, \mathcal{B})$ for this group.

Definition 4.4. Suppose that $G \stackrel{\theta}{\rightarrow} Q$ is an ordered morphism. Then its derived module is a $Q$-module $D_{\theta}$ together with a $\theta$-derivation $\delta$ such that, for each $\theta$-derivation $f: G \rightarrow \mathcal{A}$ to a $Q$-module $\mathcal{A}$, there exists a unique $Q$-map $\hat{f}: D_{\theta} \rightarrow \mathcal{A}$ with $f=\delta \hat{f}$.

Proposition 4.5. [2, Proposition 5.4.1] The derived module $D_{\theta}$ together with the derivation $G \stackrel{\delta}{\rightarrow} D_{\theta}$, of an ordered morphism $G \stackrel{\theta}{\rightarrow}$ Q exist, and $D_{\theta}$ is unique up to isomorphism.

Proof. We sketch the construction. For $e \in H_{0}$ we define

$$
X_{e}=\left\{(g, q): g \in G, q \in Q,\left(g^{-1} g\right) \theta \geqslant q q^{-1}, q^{-1} q=e\right\}
$$

and let $F^{a b}\left(X_{e}\right)$ be the free abelian group on $X_{e}$. Then we have a $Q$-module $\mathcal{F}^{a b}$ with $\left(\mathcal{F}^{a b}\right)_{e}=F^{a b}\left(X_{e}\right)$ and $\mathrm{七}(H)$-action given by $(g, q) \triangleleft(e, k)=(g, q * k)$. Note that we need the pseudoproduct here, with $q * k=\left(q \mid k k^{-1}\right) k$ in this case. We let $K_{e}$ be the subgroup of $F^{a b}\left(X_{e}\right)$ generated by all elements $(g * h, q)-(g, h \theta * q)-(h, q)$, where $g^{-1} g \geqslant h h^{-1}$ in $G_{0},\left(h^{-1} h\right) \theta \geqslant q q^{-1}$ and $q^{-1} q=e$. We set $\left(D_{\theta}\right)_{e}=F^{a b}\left(X_{e}\right) / K_{e}$, and write $\langle g, q\rangle$ for the image in $\left(D_{\theta}\right)_{e}$ of $(g, q) \in X_{e}$. Now

$[(g * h, q)-(g, h \theta * q)-(h, q)] \triangleleft(e, k)=(g * h, q * k)-(g, h \theta * q * k)-(h, q * k) \in K_{k^{-1} k}$ and so $D_{\theta}$ is a $Q$-module. The universal derivation $\delta: G \rightarrow D_{\theta}$ is given by $g \delta=$ $\left\langle g,\left(g^{-1} g\right) \theta\right\rangle$.

Example 4.6.

(a) ([2, Proposition 5.4.2]) For any ordered groupoid $G$, the augmentation ideal $K G$ is the derived module of the identity map $G \rightarrow G$.

(b) If $N$ is a union of groups, $N=\bigsqcup_{e \in N_{0}} N_{e}$, then we have a morphism $\varepsilon: N \rightarrow N_{0}$ that maps $n \in N_{e}$ to $e \in N_{0}$. The derived module of $\varepsilon$ is then the abelianisation $N^{a b}=\bigsqcup_{e \in N_{0}} N_{e}^{a b}$.

Let $\mathcal{O G}^{2}$ be the category of ordered functors between ordered groupoids: its objects are ordered functors and its morphisms are commutative squares. The construction of the derived module then defines a functor $D$ from $\mathcal{O G}^{2}$ to the category Mod of modules over ordered groupoids defined by

$$
D(G \stackrel{\theta}{\rightarrow} Q)=D_{\theta} \in \operatorname{Mod}_{Q} .
$$


The well-known correspondence between derivations and maps to semidirect products for groups can be phrased for ordered groupoids as follows.

Proposition 4.7. [2, Proposition 5.5.1] The functor D has a right adjoint $\mathcal{X}: \operatorname{Mod} \rightarrow$ $\mathcal{O G}^{2}$ defined, for $\mathcal{M} \in \operatorname{Mod}_{G}$, by $\mathcal{M} \mapsto(G \ltimes \mathcal{M} \stackrel{p}{\rightarrow} G)$

Lemma 4.8. Let $N \stackrel{\iota}{\rightarrow} G \stackrel{\varphi}{\rightarrow} Q$ be an extension of ordered groupoids. Then there is a short exact sequence of $Q$-modules $0 \rightarrow N^{a b} \stackrel{\bar{\iota}}{\rightarrow} D_{\varphi} \stackrel{\bar{\varphi}}{\rightarrow} K Q \rightarrow 0$ in which $\bar{\iota}: \bar{n} \mapsto$ $\left\langle n,\left(n^{-1} n\right) \iota \varphi\right\rangle$ and $\bar{\varphi}:\langle g, q\rangle \mapsto g \varphi * q-q$.

Proof. The exact sequence

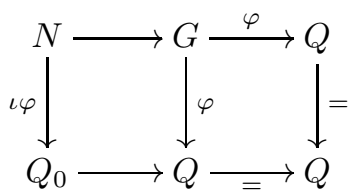

in $\mathcal{O G}^{2}$ can be rewritten as the pushout

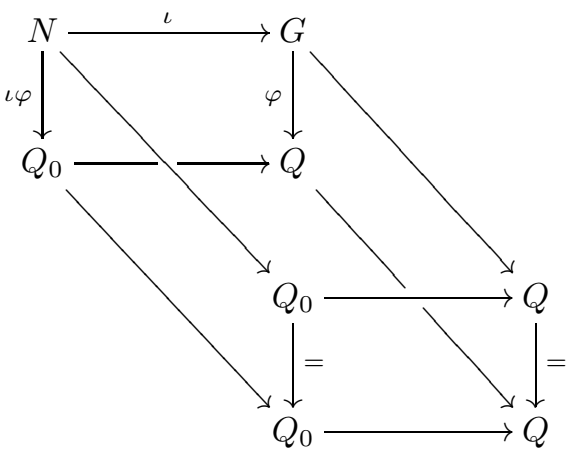

By Proposition 4.7 the functor $D$ is a left adjoint so preserves colimits. Applying $D$ to the pushout above, we deduce that the commutative square

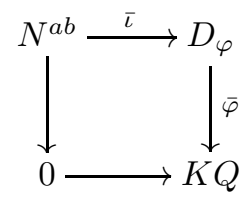

is a pushout in $\operatorname{Mod}_{Q}$, and so the sequence

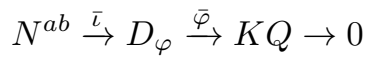

is exact. To complete the proof, we construct an abelian group homomorphism $\kappa: D_{\varphi} \rightarrow$ $N^{a b}$ such that $\bar{\iota} \kappa=$ id. We use the isomorphism $Q \cong G / / N$ from Lemma 4.2, and let $\alpha: N \rightarrow N^{a b}$ be the abelianisation. We choose a transversal $\tau: Q \rightarrow G$ to the equivalence relation

$$
g \simeq_{N} h \Longleftrightarrow \text { there exist } a, b \in N \text { such that } h=a g b .
$$

defining $G / / N$. Since $\varphi$ is identity separating, we can identify $Q_{0}$ and $G_{0}$, and take $\tau$ to be the identity on $Q_{0}$. Then for $(g, q) \in X_{e}$ (as in the proof of Proposition 4.5), we set

$$
(g, q) \kappa=\left[((g \varphi * q) \tau)^{-1} * g * q \tau\right] \alpha \in N^{a b} .
$$


Then if $g, h \in G$ with $g^{-1} g \geqslant h h^{-1}$, and $q \in Q$ with $\left(h^{-1} h\right) \varphi \geqslant q q^{-1}$ and $q^{-1} q=e$ we apply $\kappa$ to the terms in a defining relation fo $D_{\varphi}$ to find:

$$
\begin{aligned}
(g * h, q) & \stackrel{\kappa}{\mapsto}\left[((g \varphi * h \varphi * q) \tau)^{-1} * g * h * q \tau\right] \alpha, \\
(g, h \varphi * q) & \stackrel{\kappa}{\mapsto}\left[((g \varphi * h \varphi * q) \tau)^{-1} * g *(h \varphi * q) \tau\right] \alpha, \\
(h, q) & \stackrel{\kappa}{\mapsto}\left[((h \varphi * q) \tau)^{-1} * h * q \tau\right] \alpha .
\end{aligned}
$$

Comparing (4.1) with (4.2) and (4.3) we see that $\kappa$ induces an abelian group homomorphism $\kappa: D_{\varphi} \rightarrow N^{a b}$. Moreover, for $n \in N$, since $n \varphi=\left(n n^{-1}\right) \varphi$ we have

$$
\begin{aligned}
(n \alpha) \bar{\iota} \kappa & =\left\langle n,\left(n^{-1} n\right) \varphi\right\rangle \kappa=\left[(n \varphi \tau)^{-1} * n *\left(n^{-1} n\right) \varphi \tau\right] \alpha \\
& =\left[\left(\left(n n^{-1}\right) \varphi \tau\right)^{-1} * n *\left(n^{-1} n\right) \varphi \tau\right] \alpha \\
& =\left[n n^{-1} n n^{-1} n\right] \alpha=n \alpha .
\end{aligned}
$$

Hence $\bar{\imath}$ is injective, and this completes the proof of the Lemma.

Theorem 4.9. Consider an extension

$$
\mathscr{E}: N \stackrel{\mu}{\rightarrow} G \stackrel{\varphi}{\rightarrow} Q
$$

of ordered groupoids, in which $N$ is a union of groups, $\mu$ embeds $N$ as a normal ordered subgroupoid of $G$, and $Q$ is isomorphic to the quotient $G / / N$. Suppose that $\mathcal{A}$ is a $Q$ module. Then the five-term sequence

$$
0 \rightarrow \operatorname{Der}(Q, \mathcal{A}) \rightarrow \operatorname{Der}_{\varphi}(G, \mathcal{A}) \rightarrow \operatorname{Mod}_{Q}\left(N^{a b}, \mathcal{A}\right) \rightarrow H^{2}\left(Q^{I}, \mathcal{A}^{0}\right) \rightarrow H^{2}\left(G^{I}, \mathcal{A}^{0}\right)
$$

is exact.

Proof. By Lemma 4.8 we obtain the short exact sequence

$$
0 \rightarrow N^{a b} \rightarrow D_{\varphi} \rightarrow K Q \rightarrow 0
$$

of $Q-$ modules, to which we apply the apply the functor, $\operatorname{Ext}_{Q}(-, \mathcal{A})$. We obtain the exact sequence

$0 \rightarrow \operatorname{Mod}_{Q}(K Q, \mathcal{A}) \rightarrow \operatorname{Mod}_{Q}\left(D_{\varphi}, \mathcal{A}\right) \rightarrow \operatorname{Mod}_{Q}\left(N^{a b}, \mathcal{A}\right) \rightarrow \operatorname{Ext}_{Q}^{1}(K Q, \mathcal{A}) \rightarrow \operatorname{Ext}_{Q}^{1}\left(D_{\varphi}, \mathcal{A}\right)$

in low dimensions. We make the following identifications to arrive at the desired result.

By Example 4.6 (a), the augmentation module $K Q$ is the derived module of the identity map on $Q$, and its universal property then gives an isomorphism $\operatorname{Mod}_{Q}(K Q, \mathcal{A}) \cong$ $\operatorname{Der}(Q, \mathcal{A})$. Similarly $\operatorname{Mod}_{Q}\left(D_{\varphi}, \mathcal{A}\right) \cong \operatorname{Der}_{\varphi}(G, \mathcal{A})$. By Theorem 3.4 we have an isomorphism $\operatorname{Ext}_{Q}^{1}(K Q, \mathcal{A}) \cong H^{2}\left(Q^{I}, \mathcal{A}^{0}\right)$. The commutative square

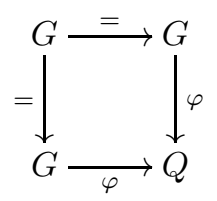

induces a map $\beta: K G \rightarrow D_{\phi}$, and so also a morphism $\operatorname{Ext}_{Q}^{i}\left(D_{\varphi}, \mathcal{A}\right) \rightarrow \operatorname{Ext}_{G}^{i}(K G, \mathcal{A})$. We show that this map is injective for $i=1$.

We proceed by embedding $\mathcal{A}$ into some injective $Q$-module $\mathcal{I}$ and set $\mathcal{C}$ to be the quotient module. Applying $\operatorname{Ext}_{G}^{*}(K G,-)$ and $\operatorname{Ext}_{Q}^{*}\left(D_{\varphi},-\right)$ to the exact sequence $\mathcal{A} \rightarrow \mathcal{I} \rightarrow \mathcal{C}$ 
gives the commutative diagram

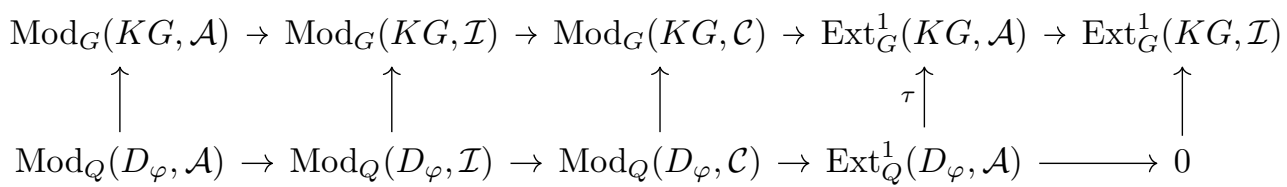

where the vertical maps are all induced by $\beta$, and we get 0 at the lower right since $\mathcal{I}$ is injective. Now any $Q$-module $\mathcal{B}$ is a $G$-module via $\varphi$, and a $Q$-map $D_{\varphi} \rightarrow \mathcal{B}$ exactly corresponds to a $\varphi$-derivation $G \rightarrow \mathcal{B}$, which is a derivation $G \rightarrow \mathcal{B}$ considering $\mathcal{B}$ as a $G$-module, and so corresponds to a $G$-map $K G \rightarrow \mathcal{B}$. Hence the first three vertical maps in the above diagram are equalities, and a simple diagram chase using exactness of the top line shows that $\operatorname{Ext}_{Q}^{1}\left(D_{\varphi}, \mathcal{A}\right) \stackrel{\tau}{\rightarrow} \operatorname{Ext}_{G}^{1}(K G, \mathcal{A})$ is injective. Making the identification $H^{2}\left(G^{I}, \mathcal{A}^{0}\right) \cong \operatorname{Ext}_{G}^{1}(K G, \mathcal{A})$ given by Theorem 3.4 we obtain an embedding $\operatorname{Ext}_{Q}^{1}\left(D_{\varphi}, \mathcal{A}\right) \hookrightarrow H^{2}\left(G^{I}, \mathcal{A}^{0}\right)$ and the sequence given in the Theorem now follows.

\section{ClassificAtion OF EXTENSIONS}

Let $\mathbb{F}(Q)$ be the free ordered groupoid on the underlying graph of the ordered groupoid $Q$. Denote the element of $\mathbb{F}(Q)$ that corresponds to an arrow $q \in Q$ by $\lfloor q\rfloor$, and define $\mathbb{F}(Q) \stackrel{\pi}{\rightarrow} Q$ by $\lfloor q\rfloor \mapsto q$. We denote its kernel by $\mathbb{N}(Q)$. If $w=\left\lfloor q_{1}\right\rfloor\left\lfloor q_{2}\right\rfloor \cdots\left\lfloor q_{m}\right\rfloor \in \mathbb{N}(Q)$ then $q_{1} \cdots q_{m} \in Q_{0}$ and so $q_{1} \mathbf{d}=q_{m} \mathbf{r}$ and so $w \mathbf{d}=w \mathbf{r}$. Therefore $\mathbb{N}(Q)$ is a union of groups.

If $\mathcal{A}$ is a $Q$-module (which we shall write additively) then it is also an $\mathbb{F}(Q)$-module via $\pi$, and so we can construct the semidirect product $S=\mathbb{F}(Q) \ltimes \mathcal{A}$. Then $T=\mathbb{N}(Q) \ltimes \mathcal{A}$ is a normal ordered subgroupoid of $S$, and since $\mathbb{N}(Q)$ acts trivially, $T$ is just the pullback $T=\{(w, a) \in \mathbb{N}(Q) \times \mathcal{A}: w \mathbf{r}=a \mathbf{d}\}$ with componentwise composition.

Now $\mathbb{F}(Q)$ acts by conjugation on $\mathbb{N}(Q)$ and, as above, on $\mathcal{A}$ via $\pi$. Hence we can consider

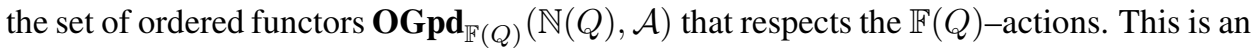
abelian group under pointwise addition in $\mathcal{A}$. Given $\phi \in \mathbf{O G p d}_{\mathbb{F}(Q)}(\mathbb{N}(Q), \mathcal{A})$, we define $M_{\phi}=\{(w, w \phi): w \in \mathbb{N}(Q)\} \subseteq \mathbb{N} \ltimes \mathcal{A}$.

Lemma 5.1. $M_{\phi}$ is a normal ordered subgroupoid of $S$ and there is an extension

$$
\mathscr{E}_{\phi}: \mathcal{A} \stackrel{\iota}{\rightarrow} S / / M_{\phi} \stackrel{\pi_{\phi}}{\longrightarrow} Q
$$

of ordered groupoids.

Proof. To show that $M_{\phi}$ is a normal ordered subgroupoid of $S$, we verify that $M_{\phi}$ satisfies the axioms NO1-NO3. The first axiom NO1 is clear.

Suppose $w \in \mathbb{N}(Q)$ and $e \in S_{0}$ such that $e \leqslant w \mathbf{d}$. Then we define the restriction of $(w, w \phi)$ to $e$ by $(e \mid(w, w \phi))=((e \mid w),(e \mid w \phi))$. However $(e \mid w) \phi=(e \phi \mid w \phi)=(e \mid w \phi)$ since $((e \mid w) \phi) \mathbf{d}=e=e \phi$ and $(e \mid w) \phi \leqslant w \phi$. Therefore

$$
(e \mid(w, w \phi))=((e \mid w),(e \mid w) \phi) \in M_{\phi}
$$

and $\mathrm{NO} 2$ holds

Let $(w, w \phi) \in M_{\phi}$ and $(h, a),(k, b) \in S$ such that $(h, a)$ and $(k, b)$ have an upper bound $(g, c) \in S$ and let $(h, a)^{-1}(w, w \phi)(k, b)$ be defined in $S$. The subgroupoid $M_{\phi}$ is a disjoint 
union of groups, and so $(h, a)^{-1}(w, w \phi)(k, b)$ being defined implies that $(h, a) \mathbf{d}=(k, b) \mathbf{d}$ : since $(h, a)$ and $(k, b)$ are restrictions of $(g, c)$ in $S$ then $(h, a)=(k, b)$. Thus

$$
\begin{aligned}
(h, a)^{-1}(w, w \phi)(k, b) & =(h, a)^{-1}(w, w \phi)(h, a) \\
& =\left(h^{-1},-a \triangleleft h^{-1}\right)(w, w \phi)(h, a) \\
& =\left(h^{-1},-a \triangleleft h^{-1}\right)(w h,(w \phi \triangleleft h)+a) \\
& =\left(h^{-1} w h,\left(-a \triangleleft h^{-1} w h\right)+(w \phi \triangleleft h)+a\right) .
\end{aligned}
$$

But $-a \triangleleft h^{-1} w h=-a$ since $h^{-1} w h \in \mathbb{N}(Q)$ acts trivially and so the second component is $w \phi \triangleleft h$, which is equal to $\left(h^{-1} w h\right) \phi$ since $\phi \in \mathbf{O G p d}_{\mathbb{F}(Q)}(\mathbb{N}(Q), \mathcal{A})$. Therefore

$$
(h, a)^{-1}(w, w \phi)(k, b)=\left(h^{-1} w h,\left(h^{-1} w h\right) \phi\right) \in M_{\phi}
$$

and NO3 holds. Therefore $M_{\phi}$ is a normal ordered subgroupoid of $S$.

We now show that $\mathscr{E}_{\phi}$ is an extension of ordered groupoids. Since $M_{\phi}$ is a normal ordered subgroupoid of $S$, the quotient ordered groupoid $S / / M_{\phi}$ exists. Define the map $S / / M_{\phi} \stackrel{\pi_{\phi}}{\longrightarrow}$ $Q$ by $[(x, a)] \mapsto x \pi$. We show that $\pi_{\phi}$ is well defined. Let $(w, a) \in S$ and suppose that $u, v \in \mathbb{N}(Q)$ and that $(u, u \phi)(w, a)(v, v \phi)$ is defined in $S$. Then we have

$$
(u, u \phi)(w, a)(v, v \phi)=(u, u \phi)(w v, a \triangleleft v+v \phi)=(u w v, u \phi \triangleleft w v+(a \triangleleft v)+v \phi)
$$

and so applying $\pi_{\phi}$ gives

$$
[(u, u \phi)(w, a)(v, v \phi)] \mapsto(u w v) \pi=w \pi=[(w, a)] \pi_{\phi} .
$$

So $\pi_{\phi}$ is well-defined, and $[w, a]_{\pi_{\phi}} \in E(Q) \Leftrightarrow w \in \mathbb{N}(Q)$. It is evident that $\pi_{\phi}$ is an ordered functor of ordered groupoids.

Now define the map $\mathcal{A} \stackrel{\iota}{\rightarrow} S / / M_{\phi}$ by $a \mapsto[(e, a)]$ for $a \in \mathcal{A}_{e}$. To show injectivity of $\iota$, let $[(f, b)] \in S / / M_{\phi}$ with $b \in A_{f}$, and suppose that $[(e, a)]=[(f, b)]$. Then for some $u, v \in \mathbb{N}(Q)$ we have

$$
(u, u \phi)(e, a)(v, v \phi)=(u e v,(u \phi \triangleleft e \triangleleft v)+a \triangleleft v+v \phi)=(f, b) .
$$

From the first component, we have uev $=f$ and so $e=f$, and $u=v^{-1}$. In the second component, $v$ acts trivially on $\mathcal{A}$ and so

$$
(u \phi \triangleleft e \triangleleft v)+a \triangleleft v+v \phi=u \phi+a+v \phi=u \phi+a-u \phi=a .
$$

Hence $a=b$ and therefore $\iota$ is injective.

We have that

$$
\begin{aligned}
\operatorname{ker}\left(\pi_{\phi}\right) & =\{(w, a): w \in \mathbb{N}(Q)\} \\
& =\left\{\left[(w, w \phi)\left(w \mathbf{r},(w \phi)^{-1} a\right)\right]\right\} \\
& =\left\{\left[\left(w \mathbf{r},(w \phi)^{-1} a\right)\right]\right\} \\
\subseteq & \operatorname{im}(\iota)
\end{aligned}
$$

Therefore $\mathscr{E}_{\phi}$ is an extension as desired.

We denote $S / / M_{\phi}$ by $G_{\phi}$, and we denote the image in $G_{\phi}$ of an element $(w, a) \in S$ by $[w, a]_{\phi}$. Note that $[w, a]_{\phi} \pi_{\phi}=w \pi$. For the trivial homomorphism $\tau \in \mathbf{O G p d}_{\mathbb{F}(Q)}(\mathbb{N}(Q), \mathcal{A})$, we have $G_{\tau}=Q \ltimes \mathcal{A}$.

Let $\mathscr{E}$ be the set of extensions constructed from the normal ordered subgroupoids $M_{\phi}$ of $S$ for $\phi \in \mathbf{O G p d}_{\mathbb{F}(Q)}(\mathbb{N}(Q), \mathcal{A})$ occurring in Lemma 5.1. Then the (abelian) group 
$\operatorname{OGpd}_{\mathbb{F}(Q)}(\mathbb{N}(Q), \mathcal{A})$ acts transitively on $\mathscr{E}:$ for $\alpha \in \mathbf{O G p d}_{\mathbb{F}(Q)}(\mathbb{N}(Q), \mathcal{A})$ we define $\mathscr{E}_{\phi} \triangleleft \alpha=\mathscr{E}_{\phi+\alpha}$.

Since $\mathbb{N}(Q)$ acts trivially on $\mathcal{A}$, when restricted to $\mathbb{N}(Q)$ any derivation $\delta: \mathbb{F}(Q) \rightarrow \mathcal{A}$ restricts to a morphism in $\mathbf{O G p d}_{\mathbb{F}(Q)}(\mathbb{N}(Q), \mathcal{A})$. This gives us the mapping

$$
\rho: \operatorname{Der}(\mathbb{F}(Q), \mathcal{A}) \rightarrow \mathbf{O G p d}_{\mathbb{F}(Q)}(\mathbb{N}(Q), \mathcal{A})
$$

in the five-term exact sequence

$0 \rightarrow \operatorname{Der}(Q, \mathcal{A}) \rightarrow \operatorname{Der}_{\pi}(\mathbb{F}(Q), \mathcal{A}) \rightarrow \operatorname{Mod}_{Q}\left(\mathbb{N}^{a b}(Q), \mathcal{A}\right) \rightarrow H^{2}\left(Q^{I}, \mathcal{A}^{0}\right) \stackrel{\varpi}{\longrightarrow} H^{2}\left(\mathbb{F}\left(Q^{I}\right), \mathcal{A}^{0}\right)$ using the fact that $\operatorname{Mod}_{Q}\left(\mathbb{N}^{a b}(Q), \mathcal{A}\right)=\operatorname{OGpd}_{\mathbb{F}(Q)}(\mathbb{N}(Q), \mathcal{A})$.

Proposition 5.2. The extensions $\mathscr{E}_{\phi}$ and $\mathscr{E}_{\psi}$ are equivalent if and only if $-\phi+\psi: \mathbb{N}(Q) \rightarrow$ $\mathcal{A}$ is the restriction of an ordered derivation $\mathbb{F}(Q) \rightarrow \mathcal{A}$.

Proof. Suppose that the extensions $\mathscr{E}_{\phi}$ and $\mathscr{E}_{\psi}$ are equivalent: that is, there exists an ordered functor $\mu: G_{\phi} \rightarrow G_{\psi}$ making the diagram

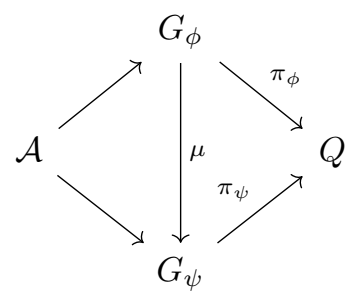

commute. Then for all $a \in A_{e}$ and $(w, b) \in S$ we have:

$$
\begin{aligned}
{[e, a]_{\phi} \mu } & =[e, a]_{\psi}, \\
{[w, b]_{\phi} \mu \pi_{\psi}=[w, b]_{\phi} \pi_{\phi} } & =w \pi=[w, b]_{\psi} \pi_{\psi} .
\end{aligned}
$$

Therefore $[w, b]_{\psi}^{-1}[w, b]_{\phi} \mu \in \operatorname{ker} \pi_{\psi}$. Setting $b=w \mathbf{r}$, we deduce that

$$
[w, w r]_{\psi}^{-1}[w, w \mathbf{r}]_{\phi} \mu=[w \mathbf{r}, a]_{\psi}
$$

for some $a \in A_{w \mathbf{r}}$. Since the map $\mathcal{A} \rightarrow G_{\psi}, a \mapsto[a \mathbf{r}, a]_{\psi}$ is injective, the element $a$ determined by equation (5.3) is unique. Hence we get a mapping $\delta: \mathbb{F}(Q) \rightarrow \mathcal{A}$ given by $w \mapsto a$, with $a \in \mathcal{A}_{w \mathbf{r}}$, and satisfying

$$
[w \mathbf{r}, w \delta]_{\psi}=[w, w \mathbf{r}]_{\psi}^{-1}[w, w \mathbf{r}]_{\phi} \mu
$$

We show that $\delta$ is an ordered derivation. Given $u, v \in \mathbb{F}(Q)$ such that the composition $u v$ exists, we have

$$
\begin{aligned}
{[v \mathbf{r},(u v) \delta]_{\psi} } & =[u v, v \mathbf{r}]_{\psi}^{-1}[u v, v \mathbf{r}]_{\phi} \mu \\
& =[v, v \mathbf{r}]_{\psi}^{-1}[u, u \mathbf{r}]_{\psi}^{-1}[u, u \mathbf{r}]_{\phi} \mu[v, v \mathbf{r}]_{\phi} \mu \\
& =[v, v \mathbf{r}]_{\psi}^{-1}[u, u \mathbf{r}]_{\psi}^{-1}[u, u \mathbf{r}]_{\phi} \mu[v, v \mathbf{r}]_{\psi}[v, v \mathbf{r}]_{\psi}^{-1}[v, v \mathbf{r}]_{\phi} \mu \\
& =[v, v \mathbf{r}]_{\psi}^{-1}[u \mathbf{r}, u \delta]_{\psi}[v, v \mathbf{r}]_{\psi}[v \mathbf{r}, v \delta]_{\psi} \quad \text { by (5.4) } \\
& =[v \mathbf{r},(u \delta) \triangleleft v+v \delta]
\end{aligned}
$$

and therefore $(u v) \delta=(u \delta) \triangleleft v+v \delta$. Hence $\delta$ is an ordered derivation $\mathbb{F}(Q) \rightarrow \mathcal{A}$. We now show that $(-\phi+\psi)$ is a restriction of $\delta$. 
Suppose that $w \in \mathbb{N}(Q)$. Then recalling that $w \mathbf{d}=w \mathbf{r}$ we have

$$
[w, w \mathbf{r}]_{\phi}=\left[(w, w \mathbf{r})\left(w^{-1}, w^{-1} \phi\right)\right]_{\phi}=\left[w \mathbf{r}, w^{-1} \phi\right]_{\phi}
$$

and so

$$
\begin{aligned}
{[w \mathbf{r}, w \delta]_{\psi} } & =\left[w \mathbf{d}, w^{-1} \psi\right]_{\psi}^{-1}\left[w \mathbf{r}, w^{-1} \phi\right]_{\phi} \mu \\
& =[w \mathbf{r}, w \psi]_{\psi}\left[w \mathbf{r}, w^{-1} \phi\right]_{\phi} \mu \\
& =[w \mathbf{r}, w \psi]_{\psi}\left[w \mathbf{r}, w^{-1} \phi\right]_{\psi} .
\end{aligned}
$$

Using [5.1), we have $[w \mathbf{r}, w \delta]_{\psi}=[w \mathbf{r}, w \psi-w \phi]$ and, since $a \mapsto[a \mathbf{r}, a]_{\psi}$ is injective, we deduce that $w \delta=w(-\phi+\psi)$. Therefore if the extensions $\mathscr{E}_{\phi}$ and $\mathscr{E}_{\psi}$ are equivalent then $(-\phi+\psi)$ is the restriction of an ordered derivation $\delta: \mathbb{F}(Q) \rightarrow \mathcal{A}$.

For the converse, let $\eta: \mathbb{F}(Q) \rightarrow \mathcal{A}$ be an ordered derivation and $\phi \in \mathbf{O G p d}_{\mathbb{F}(Q)}(\mathbb{N}(Q), \mathcal{A})$. Since $\eta$ restricts to an $\mathbb{F}(Q)$-map $\mathbb{N}(Q) \rightarrow \mathcal{A}$ and $\mathbf{O G p d}_{\mathbb{F}(Q)}(\mathbb{N}(Q), \mathcal{A})$ is closed under pointwise addition, the sum $\phi+\eta \in \mathbf{O G p d}_{\mathbb{F}(Q)}(\mathbb{N}(Q), \mathcal{A})$. Set $\psi=\phi+\eta$ and define a mapping $\nu: G_{\phi} \rightarrow G_{\psi}$ by $[w, b]_{\phi} \mapsto[w, w \eta+b]_{\psi}$. Then for $u, v \in \mathbb{N}(Q)$ we have

$$
(u, u \phi)(w, b)(v, v \phi)=(u w,(u \phi) \triangleleft w+b)(v, v \phi)=(u w v,(u \phi) \triangleleft w+b+v \phi)
$$

since $\mathbb{N}(Q)$ acts trivially on $\mathcal{A}$, and so

$$
\begin{aligned}
{[(u, u \phi)(w, b)(v, v \phi)]_{\phi} \nu } & =[u w v,(u \phi) \triangleleft w+b+v \phi]_{\phi} \nu \\
& =[u w v,(u w v) \eta+(u \phi) \triangleleft w+b+v \phi]_{\psi} \\
& =[u w v,(u \eta) \triangleleft w v+(w v) \eta+(u \phi) \triangleleft w+b+v \phi]_{\psi} \\
& =[u w v,(u \eta) \triangleleft w+w \eta+v \eta+(u \phi) \triangleleft w+b+v \phi]_{\psi} \\
& =[u w v,(u \psi) \triangleleft w+v \psi+w \eta+b]_{\psi} \\
& =[(u, u \psi)(w, w \eta+b)(v, v \psi)]_{\psi} \\
& =[w, w \eta+n]_{\psi}=[w, b]_{\phi} \nu
\end{aligned}
$$

and so $\nu$ is well-defined. It is then easy to see that $\nu$ is an ordered functor, and that

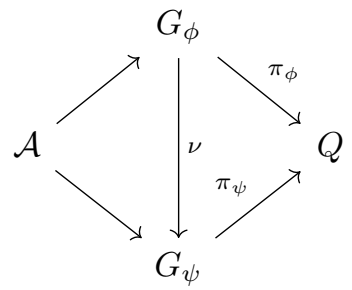

commutes, so that $\mathscr{E}_{\phi}$ and $\mathscr{E}_{\psi}$ are equivalent.

Corollary 5.3. The set of equivalence classes of extensions of $\mathcal{A}$ by $Q$ in $\mathscr{E}$ is in one-to-one correspondence with the cokernel of the restriction map

$$
\rho: \operatorname{Der}(\mathbb{F}(Q), \mathcal{A}) \rightarrow \boldsymbol{O G p d}_{\mathbb{F}(Q)}(\mathbb{N}(Q), \mathcal{A}) .
$$

Proposition 5.4. An extension $\mathcal{A} \stackrel{\iota}{\rightarrow} G \stackrel{\varphi}{\rightarrow} Q$ is equivalent to an extension $\mathscr{E}_{\alpha}$ for some $\alpha \in \boldsymbol{O G p d}_{\mathbb{F}(Q)}(\mathbb{N}(Q), \mathcal{A})$.

Proof. Lift the quotient map $\pi$ to $\pi_{*}: \mathbb{F}(Q) \rightarrow G$ making the diagram 


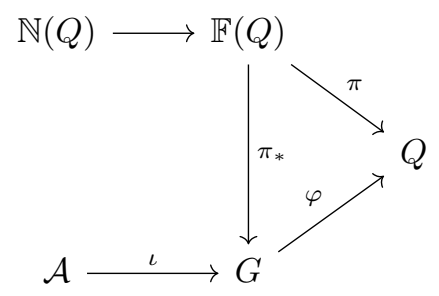

commute. Then $\mathbb{N}(Q) \pi_{*} \subseteq \mathcal{A}$ and we may define $\xi: \mathbb{F}(Q) \ltimes \mathcal{A} \rightarrow G$ by $(w, a) \xi=$ $\left(w \pi_{*}\right)(a \iota)$ and $\operatorname{ker} \xi=\left\{\left(\bar{w}, w^{-1} \pi_{*}\right): w \in \mathbb{N}(Q)\right\}$. Since each $A_{e}$ is abelian, the map $\alpha: w \mapsto w^{-1} \pi_{*}$ is a homomorphism $\mathbb{N}(Q) \rightarrow \mathcal{A}$, and $\mathscr{E}$ is equivalent to $\mathscr{E}_{\alpha}$.

Remark 5.5. In [27], Matthews discusses the concept of factor sets for modules over ordered groupoids. We note that we can recover the factor set discussed by Matthew from our construction. The restriction of the map $\pi_{*}: \mathbb{F}(Q) \rightarrow G$ to the elements $\lfloor q\rfloor^{-1}$. $\left\lfloor\left(p \mid q q^{-1}\right)\right\rfloor^{-1} \cdot\lfloor p * q\rfloor \in \mathbb{N}(Q)$ is a factor set discussed in [27].

\subsection{Cohomology and extensions of ordered groupoids.}

Theorem 5.6. The set of equivalence classes of extensions of $\mathcal{A}$ by $Q$ is in one-to-one correspondence with the second cohomology group $H^{2}\left(Q^{I}, \mathcal{A}^{0}\right)$.

Proof. From the extension $\mathbb{N}(Q) \rightarrow \mathbb{F}(Q) \stackrel{\pi}{\rightarrow} Q$ of ordered groupoids we obtain, by Lemma 4.8, the short exact sequence $\mathbb{N}^{a b}(Q) \rightarrow D_{\pi} \rightarrow K Q$ of $Q$-modules. By Theorem 4.9 and using the fact that $\operatorname{Mod}_{Q}\left(\mathbb{N}^{a b}(Q), \mathcal{A}\right)=\operatorname{OGpd}_{\mathbb{F}(Q)}(\mathbb{N}(Q), \mathcal{A})$ we obtain the fiveterm exact sequence

$$
\begin{aligned}
& 0 \rightarrow \operatorname{Der}(Q, \mathcal{A}) \rightarrow \operatorname{Der}_{\pi}(\mathbb{F}(Q), \mathcal{A}) \rightarrow \mathbf{O G p d}_{\mathbb{F}(Q)}(\mathbb{N}(Q), \mathcal{A}) \rightarrow \\
& (5.6) \\
& H^{2}\left(Q^{I}, \mathcal{A}^{0}\right) \stackrel{\varpi}{\longrightarrow} H^{2}\left(\mathbb{F}\left(Q^{I}\right), \mathcal{A}^{0}\right) .
\end{aligned}
$$

For any $Q^{I}$-module $\mathcal{B}$, a $Q^{I}$-map $K \mathbb{F}\left(Q^{I}\right) \rightarrow \mathcal{B}$ corresponds to a homomorphism $\mathbb{F}\left(Q^{I}\right) \rightarrow$ $\mathbb{F}\left(Q^{I}\right) \ltimes \mathcal{B}$. If $\mathcal{A} \rightarrow \mathcal{B}$ is an epimorphism of $Q^{I}$-modules then we obtain a lift

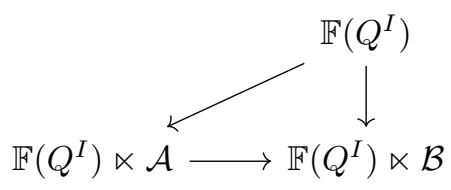

via the freeness of $\mathbb{F}\left(Q^{I}\right)$, and so we get the corresponding lift $K \mathbb{F}\left(Q^{I}\right) \rightarrow \mathcal{A}$. Thus $K \mathbb{F}\left(Q^{I}\right)$ is projective and hence the sequence $K \mathbb{F}\left(Q^{I}\right) \rightarrow \mathbb{Z} \mathbb{F}\left(Q^{I}\right) \rightarrow \Delta \mathbb{Z}$ is a projective resolution of $\Delta \mathbb{Z}$. It follows that $H^{n}\left(\mathbf{F}\left(Q^{I}\right), \mathcal{A}^{0}\right)=0$ for $n>0$, and the sequence (5.6) becomes

$$
0 \rightarrow \operatorname{Der}(Q, \mathcal{A}) \rightarrow \operatorname{Der}_{\pi}(\mathbb{F}(Q), \mathcal{A}) \rightarrow \mathbf{O G p d}_{\mathbb{F}(Q)}(\mathbb{N}(Q), \mathcal{A}) \stackrel{\varpi}{\rightarrow} H^{2}\left(Q^{I}, \mathcal{A}^{0}\right) \rightarrow 0 .
$$

Therefore $\varpi$ is a bijection from the cokernel of the restriction map $\operatorname{Der}_{\pi}(\mathbb{F}(Q), \mathcal{A}) \rightarrow$ $\operatorname{OGpd}_{\mathbb{F}(Q)}(\mathbb{N}(Q), \mathcal{A})$ to $H^{2}\left(Q^{I}, \mathcal{A}^{0}\right)$ and thus by corollary 5.3 the result follows. 


\section{REFERENCES}

[1] N. AlYamani, N. D. Gilbert, and E. C. Miller, Fibrations of ordered groupoids and the factorization of ordered functors. Appl. Categ. Structures 24 (2016) 121-146.

[2] N. AlYamani, Homological and homotopical constructions for functors on ordered groupoids. PhD Thesis, Heriot Watt University, Edinburgh, (2014).

[3] B. O. Bainson, Homology, Cohomology and Extensions of Ordered Groupoids. PhD Thesis, Heriot Watt University, Edinburgh, (2016).

[4] R. Brown, Groupoids as coefficients. Proc. London Math Soc. (3) 25 (1972) 413-426.

[5] R. Brown, P. J. Higgins and R. Sivera, Nonabelian algebraic topology. Filtered spaces, crossed complexes, cubical homotopy groupoids. With contributions by C D Wensley and S V Soloviev. EMS Tracts in Mathematics, 15. European Mathematical Society, Zürich, (2011).

[6] I. Bucur and A. Deleanu, Introduction to the theory of categories and functors. London, New York, Toronto, Sydney, Wiley-Interscience Publicatons(1968).

[7] A. Coudron, Sur les extensions des demigroupes réciproques. Bull. Soc. Roy. Sci. Liège 37 (1968) 409-419.

[8] R. H. Crowell, The derived module of a homomorphism. Adv. Math. 6 (1971) 210-238.

[9] H. D. D'Alarcao, Idempotent-separating extensions of inverse semigroups. J. Austral. Math. Soc. 9 (1969) 211-217.

[10] C. Ehresmann, Oeuvres Complète et Commentées. (A.C.Ehresmann ed.) Supplements to Cah. Topologie Géom. Différ. Catégoriques (1980-84).

[11] P. Gabriel and M. Zisman, Calculus of Fractions and Homotopy Theory. Springer Verlag (1967).

[12] N. D. Gilbert, A P-theorem for ordered groupoids. In Proc. Intl. Conf. Semigroups and Formal Languages, Lisbon 2005 J. M. André et al. (Eds.) 84-100. World Scientific (2007).

[13] N. D. Gilbert, Derivations and relation modules for inverse semigroups. Algebra Discrete Math 12 (2011) $1-19$.

[14] K. W. Gruenberg, A new treatment of group extensions. Math. Z. 102 (1967) 340-350.

[15] K. W. Gruenberg, Cohomological topics in group theory. Springer-Verlag, (1970).

[16] P. J. Higgins, Notes on categories and groupoids. Van Nostrand Reinhold Math. Stud. 32 (1971).

[17] P. J. Hilton and U. Stammbach, A Course in Homological Algebra. Graduate Texts in Math. 4. Springer Verlag, (1970).

[18] H. Lausch, Cohomology of inverse semigroups. J. Algebra. 35 (1975) 273-303.

[19] J. Leech, The $\mathcal{D}$-category of a semigroup. Semigroup Forum 11 (1975/76) 283-296.

[20] M. V. Lawson and B. Steinberg, Ordered groupoids and étendues. Cah. Topol. Géom. Différ. Catég. 45 (2004) 82-108.

[21] M. V. Lawson, Inverse semigroups: the theory of partial symmetries. World Scientific, (1998).

[22] M. V. Lawson, Left cancellative categories and ordered groupoids. Semigroup Forum 68 (2004) 458-476.

[23] M. V. Lawson, Congruences on ordered groupoids. Semigroup Forum 47 (1993) 150-167.

[24] M. Loganathan, Cohomology of inverse semigroups. J. Algebra 70 (1981) 375-393.

[25] M. Loganathan, Cohomology and extensions of regular semigroups. J. Austral. Math. Soc. (Ser. A) 35 (1983) 178-193.

[26] S. MacLane, Categories for the Working Mathematician. 2nd Ed. Graduate Texts in Math. 5 Springer Verlag (1998).

[27] J. Matthews, Topological Ideas in Inverse Semigroup Theory. PhD Thesis, University of Wales, Bangor, (2004).

[28] B. Steinberg, Factorization theorems and morphisms of ordered groupoids and inverse semigroups, Proc. Edin. Math. Soc. 44 (2001) 549-569.

[29] P. J. Webb, An introduction to the representations and cohomology of categories. In Group Representation Theory, M. Geck, D. Testerman, J. Thévenaz (Eds.) EPFL Press Lausanne (2007) 149-173.

[30] P. J. Webb, Resolutions, relation modules and Schur multipliers for categories. J. Algebra 326 (2011) 245276

[31] A. Weinstein, Groupoids: unifying internal and external symmetry. Notices Amer. Math. Soc. 43 (1996) 744-752.

[32] F. Xu, Representations and Cohomology of Finite Categories. Lecture notes, Universitat Autònoma de Barcelona, www.mat.uab.cat/ xu/CatRepCoh.pdf (2011). Accessed February 2017. 